\title{
WIRELESS MESH NETWORKS PLANNING BASED ON PARAMETERS OF QUALITY OF SERVICE
}

\author{
Marlon da Silva ${ }^{1}$, Edson Luiz França Senne ${ }^{2}$ and Nandamudi Lankalapalli Vijaykumar ${ }^{1}$ \\ ${ }^{I}$ National Institute of Spatial Researches, São José dos Campos, Brazil \\ ${ }^{2}$ Department of Mathematics, UNESP, Guaratinguetá, Brazil \\ \{marlon.silva,vijay\}@lac.inpe.br,elfsenne@feg.unesp.br
}

Keywords: Wireless mesh networks; QoS; Mathematical Programming; Monte Carlo simulation.

\begin{abstract}
The use of QoS parameters to evaluate the quality of service in a mesh network is essential mainly when providing multimedia services. This paper proposes an algorithm for planning wireless mesh networks in order to satisfy some QoS parameters, given a set of test points (TPs) and potential access points (APs). Examples of QoS parameters include: probability of packet loss and mean delay in responding to a request. The proposed algorithm uses a Mathematical Programming model to determine an adequate topology for the network and Monte Carlo simulation to verify whether the QoS parameters are being satisfied. The results obtained show that the proposed algorithm is able to find satisfactory solutions.
\end{abstract}

\section{INTRODUCTION}

The extensive use of portable devices, such as laptops and mobile telephones, has contributed to a significant increase of the use of wireless mesh networks (WMNs). In these networks, the main applications use multimedia (audio and video) services. Therefore, such services must be provided as readily as possible, in order to avoid delays which could compromise the quality of service offered to the clients. In order to achieve a good quality of service, a WMN must be well planned.

The main characteristic of a WMN is the multi hop transmission, where the data is transmitted from one network device to other devices, extending the network coverage its clients. Such architecture offers Internet access at locations where it is difficult to install an infrastructure needed to directly feed the access points (APs) by an external network.

The planning of a WMN involves finding an appropriate set of equipment that composes the mesh, because an improper installation of the network may result in either unnecessary costs or in a structure unable to attend the clients satisfactorily.

However, beyond being well structured, a WMN must also offer a good quality service to its clients. Here, it is common to use some parameters to assess if the network has a desired performance level - the quality of service (QoS) parameters.

This paper focuses in the problem of finding, at the lowest cost, a topology for WMNs that satisfies all client's requirements within a quality of service previously set, with the smallest possible number of network devices.

To attend these objectives, initially a set of APs, that have to be used and obeying network coverage constraints, are determined by employing a Mixed Integer Linear Programming (MILP). Then, Monte Carlo simulation (MCS) is used to determine the values of the QoS parameters: probability of packet loss and the average delay. If the value obtained for a QoS parameter is not within the expected interval, the algorithm will suggest a modification in the WMN topology and the verification of QoS parameters will be applied again. This process continues until all parameters are attended.

The paper is organized as follows. In Section 2, there are some related works about WMN planning, QoS parameters and methods that will be used MILP and MCS. Section 3 shows how the algorithm, presented in this paper, is organized and the methods used to find a satisfied topology for WMN - a MILP, followed by MCS to verify QoS parameters. Numerical results are given in Section 4. Conclusions and future research are described in Section 5. 


\section{DEFINITIONS}

\subsection{Network Topology Problem}

WMNs (Akyildiz et al., 2005; Silva et al., 2010) are networks composed by APs (Access Points) and TPs (Test Points or Clients). APs compose the main structure of the WMN and classified as: routers responsible only for sending data to TPs - and gateways - feed the network, using external data sources - usually are installed in places with high visibility.

Figure 1 shows an example of a WMN, showing the main elements that compose it (a) and one AP installed on the top of a building located at UFPA (Federal University of Pará, Belém, Brazil) (b), where the router is inside the box in the center of antenna that amplifies the signal for increasing the coverage ratio.
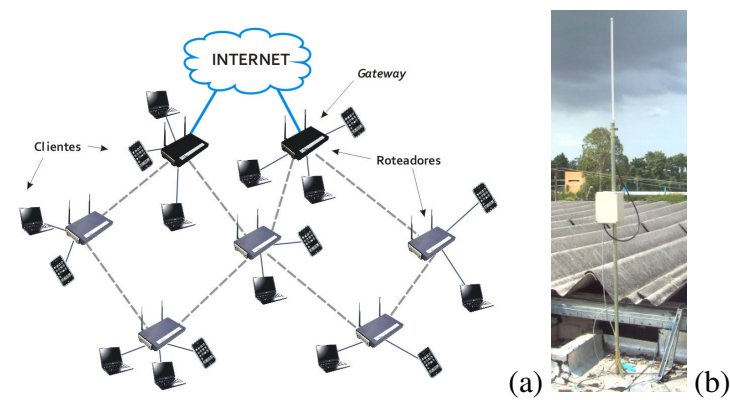

Figure 1: (a) Wireless mesh network sample (Silva et al., 2010) and (b) a mesh antenna

WMNs (Lee et al., 2006) are being intensively used in residences, buildings, universities, companies and vehicles and can deal with applications that cannot be supported by other wireless technologies, because they have as main advantages the extended coverage, strength, selfconfiguration, easy maintenance and low cost and may be installed in areas with large coverage, where it is difficult to install cables and conductors, as in locations found in emergency services.

The functional characteristics of a WMN are illustrated in Díaz and Díaz (2006), where difficulties that can be found in a wireless network, such as transmission capacity that decreases in function of the distance between the router and the client; channel interferences, where only three channels can overlap in a region; and objects that may interfere in the wireless transmission.

In order to install a WMN that can attend all the requirements of the clients, good planning is necessary, so that the network can serve all customers as best as possible, avoiding lack of quality in the network and possible waste of equipment.

WMN planning is studied in Benyamina et al. (2008), Amaldi et al. (2008) and Cabral and Mateus (2009) differently from other networks, because WMNs are planned geographically, where the position and the configuration of APs depend only on the conditions of local connectivity between clients and closer network devices. Given a set of candidate points to install APs and a set of clients, some MILP models were proposed, whose objective was to minimize the total cost of installation, taking into account both the requirements of location and multihop connectivity.

\subsection{Evaluating QoS Parameters}

Some QoS parameters, such as the capacity of assuring service for some traffic types, by means of some available technologies are defined in Abelém et al. (2007). The main characteristics of QoS in a WMN are listed, which are adopted as metrics to measure how good the service is provided by the network.

Performance measures are applied primarily to attend demands for multimedia packages (audio and video). In Saade et al. (2007) and Abelém et al. (2007), some communication protocols in WMNs are presented, which are basically divided into two types: proactive (routes already determined) and reactive (on demand). Other protocols have been used, such as adaptive and hybrid, combining main characteristics of proactive and reactive protocols, and is ideal for large networks containing a large amount of devices, such as WMN.

In a network planning where the metrics of QoS must be considered, it is necessary to verify the quality of the services offered. To do this, computational or mathematical models can be used to measure QoS parameters. Simulation is one of the most used techniques to represent and evaluate a real scenario.

\subsection{Monte Carlo Simulation}

Monte Carlo Simulation (MCS) (Mun, 2006) is often used to predict possible risks that a system may suffer. It is more efficient than other methods because it uses the initial parameters that direct the system behaviour. By the occurrence of events which behave according to probability distributions (Rubinstein and Kroese, 2007), it is possible to easily extract measures of a high complex real 
model. Random demands, based on the probability distribution that best represents its operation, stimulate the functioning of the system and provide some performance measures.

An example of this type of application is shown in Atkinson et al. (2008), where the authors proposed an algorithm for MCS applied to a system of emergency service.

\section{THE PROPOSED MODEL}

The model discussed in this paper seeks a low cost topology of a WMN, provided that QoS parameters - loss probability $\left(p_{\text {loss }}\right)$ and average delay $(D)$ - are satisfied. These values must be less than or equal to expected values for the network $\left(p_{\text {expec }}\right.$ and $\left.D_{\text {expec }}\right)$.

Figure 2 illustrates the steps of the algorithm proposed to obtain a network topology which minimizes installation cost and satisfies QoS parameters.

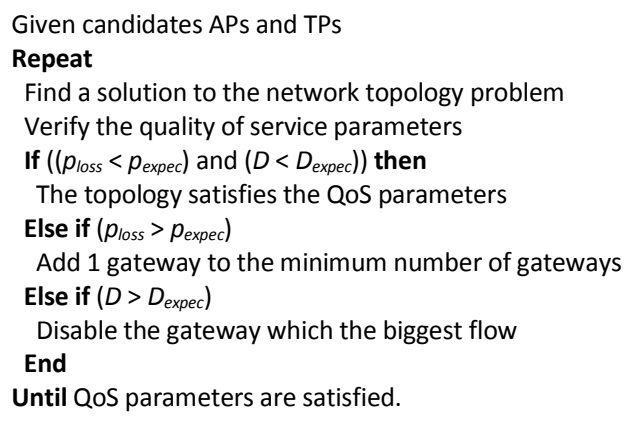

Figure 2: Algorithm to obtain the solution of model

Given the set $P=\{1,2, \ldots, n\}$ of candidate APs and the set $C=\{1,2, \ldots, m\}$ of TPs, it is possible to calculate the matrices of distances from AP to TP and from AP to AP. These matrices will be used to guarantee the network coverage.

\subsection{Network Topology Problem}

Initially, one solution must be found to the network topology problem by solving a MILP model adapted by idea presented in Benyamina et al (2008) and Amaldi et al. (2008). The difference presented in this work is in constraints that reduce the amount of variables and in establishing a minimum of gateways.

Given the values to the parameters previously established (Table 1), the solution of this model shows a network topology with lowest cost, through the values of active APs $\left(z_{j}\right)$, gateways $\left(g_{j}\right)$, links between APs $\left(y_{j l}\right)$, links between TPs and APs $\left(x_{i j}\right)$ and flow variables to links between APs $\left(f_{j l}\right)$ and gateways $\left(F_{j}\right)$, with $i \in C$ and $j, l \in P$.

Table 1: MILP Parameters

\begin{tabular}{|l|c|}
\hline Description & Parameter \\
\hline AP instalation cost & $S_{j}$ \\
\hline Additional installation cost of a gateway & $K_{j}$ \\
\hline Covering between APs $(=1$, if $j$ covers $l)$ & $b_{j l}$ \\
\hline Covering TP-AP $(=1$, if $j$ covers $i)$ & $c_{i j}$ \\
\hline Initial demand of a TP $i$ & $h_{i}$ \\
\hline Link capacity between APs & $u_{j l}$ \\
\hline Service capacity of AP & $v_{j}$ \\
\hline Traffic capacity of a gateway & $M$ \\
\hline Initial number of gateways & $G$ \\
\hline
\end{tabular}

The MILP model is as follows:

Minimize $\sum_{j=1}^{n}\left(S_{j} z_{j}+K_{j} g_{j}\right)$

Subject to

$$
\begin{array}{lr}
\sum_{j=1}^{n} x_{i j}=1 & \forall i \in C \\
x_{i j} \leq z_{j} c_{i j} & \forall i \in C, \forall j \in P \\
\sum_{i=1}^{m} h_{i} x_{i j}+\sum_{l=1}^{n}\left(f_{l j}-f_{j l}\right)-F_{j}=0 & \forall j \in P \\
f_{j l} \leq u_{j l} y_{j l} & \forall j, l \in P \\
\sum_{i=1}^{m} h_{i} x_{i j} \leq w_{j} & \forall j \in P \\
F_{j} \leq M g_{j} & \forall j \in P \\
\sum_{j=1}^{n} g_{j} \geq G & \\
2 y_{j l} \leq b_{j l}\left(z_{j}+z_{l}\right) & \forall j, l \in P \\
g_{j} \leq z_{j} & \forall j \in P \\
\sum_{l=1}^{n}\left(y_{j l}+y_{l j}\right) \leq 3 & \forall j \in P \\
x_{i j}, y_{j l}, z_{j}, g_{j} \in\{0,1\} & \forall i \in C, j, l \in P
\end{array}
$$

In this model, the objective function (1) minimizes the cost of installation of APs $(S j)$ and the additional cost of installation of gateways $(K j)$ in the WMN. The guarantee that a TP has been linked in a $\mathrm{AP}$ is described in constraint (2). Inequation (3) guarantees coverage between $\mathrm{AP} j$ and TP $i$. Constraint (4) defines the flow balance of each AP. There are constraints to flow capacity on each link (5), on each AP (6) and each gateway (7) on network. The minimum number of gateways 
(denoted by $G$ ) required in the network is described in constraint (8). Constraint (9) ensures that there is at least one link between two active APs, since these points are covered together. Inequation (10) ensures that a gateway must be an active AP of the network. An AP must contain at the most three links, due to maximum amount of channels in a wireless network, described in constraint (11) (Díaz and Díaz, 2006).

The optimal solution to this Mathematical Programming model shows the values of binary variables of links between AP and TP $\left(x_{i j}\right)$, links between APs $\left(y_{j l}\right)$, state of AP activity $\left(z_{j}\right)$ and use of gateways $\left(g_{j}\right)$.

\subsection{Verification of QoS parameters}

Results obtained from Mathematical Programming model are used to verify QoS parameters of the network. This is obtained by Monte Carlo simulation based on Atkinson et al. (2008). The objective of Monte Carlo simulation is to obtain values of loss probability and average delay for three data types common data, audio and video. Some rates are used to represent the traffic of network.

The difference to original model is in the addition of multihop application over behavior of the system and in evaluating system response delay within the simulation.

Let $\lambda_{d i}$ be the demand rate to data, $\lambda_{a i}$ the demand rate to audio and $\lambda_{v i}$ the demand rate to video, for all $\mathrm{TP} i \in C$ and $\mu_{j}$ the service rate of $\mathrm{AP} j \in P$. Let $\xi=\left[\xi_{1}, \xi_{2}, \ldots, \xi_{n}\right]$ be the vector to control the amount of packets within of each candidate AP. If candidate AP $j$ has been disabled, then its respective value for $\xi_{j}$ is equal to 0 . Otherwise, either $\xi_{j}$ maybe is greater than zero or less than the maximum capacity of service queue, denoted by $\Gamma_{j}$.

Let $\Theta^{(k)}$ be the total service rate of all calls in the system at the event $k$, initialized by 0 and $\Lambda=\sum_{i=1}^{m}\left(\lambda_{\mathrm{di}}+\lambda_{\mathrm{ai}}+\lambda_{\mathrm{vi}}\right)$ the total of demand rates across network.

To obtain values for $p_{\text {loss }}$ and $D$, the simulation algorithm follows steps below, given $T=(d, a$ or $v)$, where $d$ represents data, $a$ represents audio and $v$ represents video packets:

1) Simulate random variable $\omega \in[0,1]$.

2) If $\omega \leq \Lambda /\left(\Lambda+\Theta^{(k)}\right)$, then enter a request in a network, $\gamma_{T}^{(k+1)}=\gamma_{T}^{(k)}+1$ if have been a request of type $T$. Simulate random variable $\sigma \in C$, which probability is equal to $\lambda_{T \sigma} / \Lambda$ for every type $T$. If $\xi_{\sigma}^{(k)} \leq \Gamma_{\sigma}$, then request enters to the queue and $\xi_{\sigma}^{(k+1)}=\xi_{\sigma}^{(k)}+1$ in the random instant $\psi^{(k)}$. Else, packet has been lost, $\gamma_{T, \text { loss }}^{(k+1)}=\gamma_{T, \text { loss }}^{(k)}+1$ if lost packet is a request of type $T$.

$3)$ If $\omega>\Lambda /\left(\Lambda+\Theta^{(k)}\right)$, then simulate random variable $\delta \in P$, with probability $\theta_{\delta}^{(k)} / \Theta^{(k)}$, such that $\theta_{\delta}^{(k)}=\xi_{\delta}^{(k)} \times \mu_{i}$ if $\xi_{\delta}^{(k)}>0$. Then, $\xi_{\delta}^{(k+1)}=\xi_{\delta}^{(k)}-1$ and, for the next AP of the route $\varphi \in P, \quad \xi_{\varphi}^{(k+1)}=\xi_{\varphi}^{(k)}-1$. But, if $\varphi$ is a gateway, then packet is attempt and, for $\eta$ is the number of hops that packet used to travel until a gateway to be attempt, average delay is given as $D_{T}^{(k)}=\left(\psi_{T}^{(k)}-\psi_{T}^{(k-1)}\right) / \eta$ for every type $T$.

4) For some $j \in P$, then calculate $\Theta^{(k+1)}=\sum_{j: \xi j i j} \mu_{j}$ and $\psi^{(k+1)}=\psi^{(k)}+1$. Repeat step 1 until $N$ iterations.

Completed the simulation, QoS parameters is estimated, loss probability is equal to $p_{T, \text { loss }}=\gamma_{T, \text { loss }}^{(k)} / \gamma_{T}^{(k)}$ and average delay is equal to $D_{T}=\sum_{k=1}^{N} D_{T}^{(k)} / N$ for every type of information $\mathrm{T}$.

Values obtained in the simulation are compared with ideal values for loss probability $\left(p_{\text {expec }}\right)$ and average delay $\left(D_{\text {expec }}\right)$.

If $p_{\text {loss }}$ is less than $p_{\text {expec }}$ and $D$ is less than $D_{\text {expec }}$, then the topology found by MILP model is good and algorithm is finished. Else if $p_{\text {loss }}$ is greater than $p_{\text {expec }}$ then one gateway will be added to the minimum amount of gateways $G$ (constraint (8) of the model) and will find a new solution for network topology. Else if $D$ is greater than $D_{\text {expec }}$, then the higher value of flow variable $F_{j}, j \in P$ will be examined. For gateway $j$ that has the largest flow to external network, will be added on model a constraint that determines this point will be not more a gateway:

$$
g_{j}^{(k+1)} \leq 0 .
$$

The MILP model with added constraint will be providing a new topology to network.

\section{COMPUTATIONAL RESULTS}

The algorithm is coded in C programming language and all the experiments were carried out on a AMD 2.6 GHz. MILP run by CPLEX 12.1 and MCS is implemented 100 times, whose result is based on arithmetic mean. Two scenarios with these configurations were tested. 


\subsection{Scenario 1}

The algorithm to solve the problem of WMN planning was implemented on a scenario based on a residential area located at Pindamonhangaba, Brazil. The points used are distributed based on the problem studied in Benyamina et al. (2008).

This scenario, Scenario 1, has a square area of $360000 \mathrm{~m}^{2}$, with $n=49$ AP candidate points, disposed on a grid and $m=150$ TPs, with random coordinates. Figure 3 shows the location of all points. Initial values are as follows: $\left(h_{i}: 2 \mathrm{Mb} / \mathrm{s}\right.$, $u_{j l}: 54 \mathrm{Mb} / \mathrm{s}, w_{j}: 54 \mathrm{Mb} / \mathrm{s}, M: 128 \mathrm{Mb} / \mathrm{s}, S_{j}: 200, K_{j}: 8^{*} S_{j}$, $G: 3)$ and service rates $\left(\mu_{i}: 2 \mathrm{Mb} / \mathrm{s}\right)$ and demand rates are random.

The expected QoS parameters to the network are: $5 \%$ for data, $1 \%$ for audio and $1 \%$ for video. The average delay allowed for the network is $100 \mathrm{~ms}$ for audio and $100 \mathrm{~ms}$ for video.

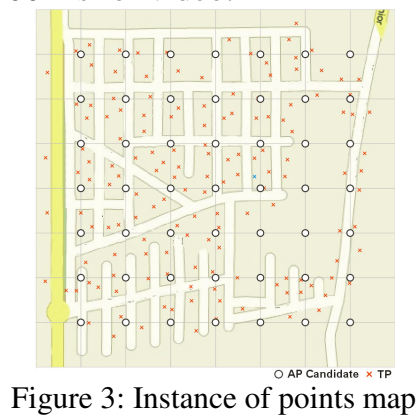

Table 2 illustrates, for each algorithm iteration, the values of objective function, represented by network installation cost, the amount of APs used in the network and QoS values for loss probability and average delay.

Table 2: Results found during algorithm execution

\begin{tabular}{|c|c|c|c|c|c|c|c|c|}
\hline \multirow{2}{*}{ It. } & \multirow{2}{*}{ Cost } & \multirow{2}{*}{ APs } & \multirow{2}{*}{ G } & \multicolumn{4}{|c|}{ P loss } & \multicolumn{2}{|c|}{ D (ms) } \\
\cline { 5 - 9 } & & & & $\boldsymbol{d}$ & $\boldsymbol{a}$ & $\boldsymbol{v}$ & $\boldsymbol{a}$ & $\boldsymbol{v}$ \\
\hline 1 & 7600 & 13 & 3 & 0.0759 & 0.0167 & 0.0162 & 81 & 79 \\
\hline 2 & 9000 & 13 & 4 & 0.0069 & 0.0000 & 0.0002 & 19 & 65 \\
\hline
\end{tabular}

Note that in the first iteration, the loss probability for data, audio and video for the topology found by Mathematical Programming model (Figure 4a) are low values, close to those tolerable by the network, but still above the allowed values.

After adding one gateway to the network, the values of QoS parameters loss probability and average delay have significantly decreased, mainly for audio loss packet, which decreased to 0 . Figure 5 can be seen as the network topology that attends the QoS parameters.

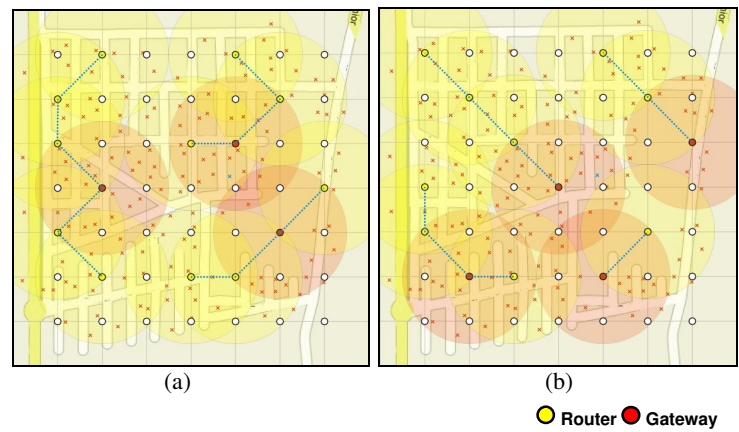

Figure 4: (a) Initial solution from MP model and (b) final solution for Scenario 1

Computational time of MILP decreases from 6445.65 to $72.54 \mathrm{~s}$. This occurs due to increase in the minimum number of gateways, which allows the program to find quickly the optimal solution. Computational time of MCS decreases from 379 to 219 s.

\subsection{Scenario 2}

Scenario 2 has a square area of $160000 \mathrm{~m}^{2}$, with $n=25$ AP candidate points, with random coordinates and $m=80$ TPs, with random coordinates. Figure 5 shows the location of all points. The initial values adopted by Benyamina et al. (2008) and used to solve the network coverage problem and to QoS verification are as follows: $\left(h_{i}: 2 \mathrm{Mb} / \mathrm{s}, \quad u_{j l}: 54 \mathrm{Mb} / \mathrm{s}, \quad w_{j}: 54 \mathrm{Mb} / \mathrm{s}, \quad M: 128 \mathrm{Mb} / \mathrm{s}\right.$, $S_{j}: 200, K_{j}: 8 * S_{j}, G: 1, \mu_{i}: 2 \mathrm{Mb} / \mathrm{s}, \lambda_{d i}: 0 \sim 300 \mathrm{~kb} / \mathrm{s}, \lambda_{a i}:$ $\left.0 \sim 700 \mathrm{~kb} / \mathrm{s}, \lambda_{v i}: 0 \sim 1 \mathrm{Mb} / \mathrm{s}\right)$.

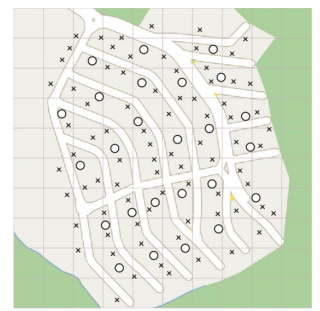

Figure 5: Instance of points map

Table 3 illustrates, for every iteration of the algorithm, the values of objective function (network installation cost), the amount of APs used in the network and QoS values for loss probability and average delay where expected QoS parameters to the network are: $15 \%$ for data, $10 \%$ for audio and $10 \%$ for video and average delay allowed for the network is $150 \mathrm{~ms}$ for audio and $150 \mathrm{~ms}$ for video. 
Table 3: Results found during algorithm execution

\begin{tabular}{|c|c|c|c|c|c|c|c|c|}
\hline \multirow{2}{*}{ It. } & \multirow{2}{*}{ Cost } & \multirow{2}{*}{ APs } & \multirow{2}{*}{$\mathbf{G}$} & \multicolumn{4}{|c|}{ p $_{\text {loss }}$} & \multicolumn{2}{|c|}{$\mathbf{D}$ (ms) } \\
\hline & & & & $\boldsymbol{d}$ & $\boldsymbol{a}$ & $\boldsymbol{v}$ & $\boldsymbol{a}$ & $\boldsymbol{v}$ \\
\hline 1 & 1600 & 8 & 1 & 0.2754 & 0.2330 & 0.2286 & 134 & 142 \\
\hline 2 & 2400 & 8 & 2 & 0.1212 & 0.0699 & 0.0654 & 147 & 152 \\
\hline 3 & 3100 & 7 & 2 & 0.0671 & 0.0116 & 0.0076 & 142 & 148 \\
\hline
\end{tabular}

In the first iteration, the model found a topology that covers the area totally (Figure 6a), but it is not ideal. Note in the second iteration, the loss probability for data, audio and video for the topology found by MCS obtain allowed values. However, the average delay to network for video is not satisfied and, reallocating the gateway with biggest flow, the algorithm obtained a good topology in the third iteration (Figure 6b).

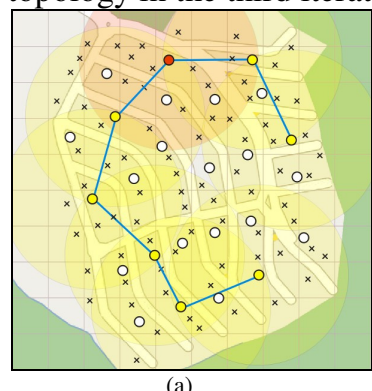

(a)

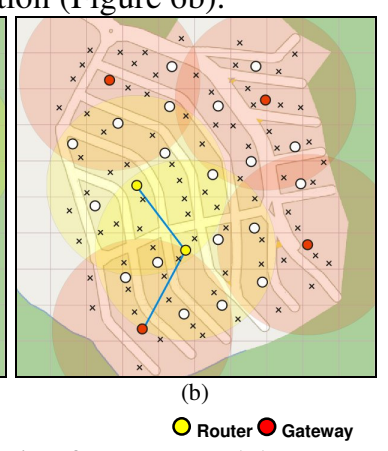

Figure 6: Initial solution from MP model
Computational time of MILP decreases from 76.69 to $10.02 \mathrm{~s}$, while the time of MCS does not change, maintaining 130 s per iteration.

\section{CONCLUSIONS}

This paper presented an alternative method for WMN planning which aims to find a low cost topology satisfying some QoS values for the network. Simulation of the operation of WMNs through MCS is a very effective method to preview the network performance.

It is intended, as a future work, to use other methods to evaluate network performance, besides using heuristics to find a satisfactory solution to Mathematical Programming model, as comparative mode with this model and other models, such as Queuing Networks and Stochastic Programming.

\section{ACKNOWLEDGEMENTS}

M. Silva acknowledges National Council for Scientific and Technological Development from Brazil $(\mathrm{CNPq})$ for the financial support.

\section{REFERENCES}

Abelém, A. J. G., Albuquerque, C. V. N., Saade, D. C. M., Aguiar, E. S., Duarte, J. L., Fonseca, J. E. M., Magalhães, L. C. S., 2007. Redes Mesh: mobilidade, qualidade de serviço e comunicação em grupo. Minicourses Book of SBRC 2007. Belém: cap. 2.

Akyildiz, I. F., Wang, X., Wang, W., 2005. Wireless mesh networks: a survey, Computer Networks, vol. 47, pp. 445-487.

Amaldi, E., Capone, A., Cesana, M., Filippini, I., Malucelli, F., 2008. Optimization Models and Methods for Planning Wireless Mesh Networks. Computer Networks, vol. 52, pp. 2159-2171, 2008.

Atkinson, J. B., Kovalenko, I. N., Kuznetsov, N., Mykhalevych, K. V., 2008. A hypercube queueing loss model with customer-dependent service rates. European Journal of Operational Research, vol. 191, pp. 223-239.

Benyamina, D., Hafid, A., Gendreau, M., 2008. On the Design of Bi-connected Wireless Mesh Network Infrastructure with QoS Constraints, IEEE GLOBECOM, pp. 5307-5312.

Cabral, G. A., Mateus, G. R., 2009. A approach based on simulation to wireless mesh networks planning, Proceedings of XLI Brazilian Simposium of Operational Research (SBPO), Porto Seguro: UNIFACS.

Chase, R. B., Jacobs, F. R., Aquilano, N. J., 2004. Operations Management for Competitive Advantage. 10 ed. New York: McGraw Hill.

Díaz, L. E. N., Díaz, J. A. P., 2006. A Model for designing WLAN's 802.11 for VoIP. Proceedings of Electronics, Robotics and Automotive Mechanics Conference, Guernavaca: IEEE.

Lee, M. J., Zheng, J., Ko, Y. B., Shrestha, D. M., 2006. Emerging standards for wireless mesh technology. IEEE Wireless Communications, v. 13, n.2, p. 56-63.

Mun, J., 2006. Modeling risk: applying Monte Carlo simulation, real options analysis, forecasting, and optimization techniques. New York: John Wiley \& Sons.

Rubinstein, R. Y., Kroese, D. P., 2007. Simulation and the Monte Carlo method. New York: John Wiley Inc.

Saade, D. C. M., Albuquerque, C. V. N., Magalhães, L. C. S., Passos, D. Duarte, J., Valle, R., 2007. Mesh networks: lower cost solution to popularization of Brazilian Internet access. Proceedings of Brazilian Simposium of Telecommunications (XXV SBrT). Recife.

Silva, M., Senne, E. L. F., Vijaykumar, N. L., 2010. Wireless mesh networks planning with Hypercube queueing model to verifying of QoS parameters. Proceedings of XLII Brazilian Simposium of Operational Research, Bento Gonçalves: UFSM. 